Manuscript article to be published Engineering of Scintillation Materials and Radiation Technologies, M. Korzhik and A. Gektin (eds.), in: Springer Proceedings in Physics2018

\title{
Calorimeter designs based on fibre-shaped scintillators
}

\author{
K. Pauwels', M. Lucchini', A. Benaglia', E. Auffray ${ }^{2}$ \\ University of Milano-Bicocca, Piazza dell'Ateneo Nuovo 1, Milano, 20125, Italy \\ European Organization for Nuclear Research, Geneva 23, Switzerland \\ Princeton University, Princeton, NJ, 08544 USA
}

\begin{abstract}
Crystal fibres of high density represent a flexible and powerful tool for the design of calorimeters capable to operate under the challenging environments of future accelerator experiments. The high light yield and good radiation tolerance of garnet crystal fibres allow using them as active detecting elements for detectors operating in harsh radiation environments such as those of high luminosity hadron colliders. Recent improvements in the material engineering have also demonstrated the possibility to reduce the scintillation decay time constant of garnet crystals such as LuAG, YAG and GAGG by addition of divalent ions. This makes garnet materials even more suitable for applications where the radiation detection occurs at very high rates. In the following we summarize the progress made on both technology development and detector design achieved in the past years with the goal of tailoring crystal fibres for future calorimetry applications.
\end{abstract}

\section{Introduction}

Calorimeters at future colliders will have to face challenging operation conditions such as harsh radiation levels and high rate as for example in the case of High Luminosity LHC where up to an average of 200 collisions are expected every $25 \mathrm{~ns}$ [1]. This will set stringent requirements on the detectors which have to be constructed of radiation tolerant components, and be capable of providing high spatial granularity and good time resolution in order to reduce the effect of event pile up.

While operating under these conditions, High Energy Physics (HEP) detectors must guarantee the precise measurement of all the particles produced during collisions including hadrons and jets. One example is the need to separate heavy bosons, $\mathrm{W}$ and $\mathrm{Z}$, by reconstructing their invariant masses in di-jet final states, which requires significant improvements in jet energy resolution with respect to today's systems. This is mostly, but not exclusively, addressed in the context of detectors for the High Luminosity upgrade of the Large Hadron Collider at CERN (HL-LHC) as well as for future electron positron linear colliders (ILC [2], CLIC [3]) and future circular collider (FCC [4]), where performance requirements drive the R\&D for 
highly granular and radiation hard detectors. The hadronic energy resolution of today's calorimeters is at best $50 \% / \sqrt{ } \mathrm{E}$ and is fundamentally limited by intrinsic eventto-event fluctuations of the shower development, which is characterized by a large variety of physics processes and relatively low particle statistics.

To improve the performances of future calorimeters, two main directions are being followed, which both take an integrated approach at electromagnetic and hadronic calorimetry. They are complementary and can ultimately be combined.

- The particle flow approach (PFA) aims at reconstructing each particle in a jet individually and thus optimizing the jet energy resolution by combining tracking and calorimetric measurements. It minimizes the impact of the hadronic resolution to the small fraction of neutral particles in a jet. Particle flow calorimeters need excellent imaging capabilities and must therefore be very finely segmented in the three dimensions [5].

- The dual readout approach aims at improving the hadronic energy resolution itself by simultaneously measuring the total deposited energy by means of scintillation light and the electromagnetic (relativistic) component by means of Cherenkov light. This allows correcting for fluctuations of the shower composition event-by-event, which otherwise limit the resolution if the calorimeter is noncompensating [6].

We proposed an alternative to these approaches, which has the potential of combining their relative merits and limiting their drawbacks [7-8], based on inorganic crystals of high density. By selecting scintillating materials activated by a doping ion, instead of self-activated scintillators, both doped and undoped fibres of a same material could be mixed. These materials would then behave respectively as scintillators and Cherenkov radiators [9]. The active part of the obtained detector is then dense enough to allow compact designs. Besides, this system is capable of disentangling the electromagnetic and non-electromagnetic components of a shower with a uniform radiation length, Moliere radius and interaction length.

The progress made in the micro-pulling down technology (see [10] for a review of some of the achieved results) encouraged us to consider fiber-shaped geometries to gain flexibility in the choice of detector geometry and its spatial granularity. Garnet crystals, such as LuAG, YAG and GAGG, doped with Cerium are good candidates as they were proven to be radiation tolerant up to very high levels of both ionizing radiation and hadron fluences [11]. They can also be engineered by addition of divalent ions to optimize their scintillation kinetics and make them suitable for high rate applications. Faster decay constants were for instance obtained on LuAG:Ce crystals when codoped with $\mathrm{Mg}^{2}$ ions [12]. Similar techniques also produce faster scintillation in other garnet crystals [13]. 


\section{Calorimeter designs}

As illustrated in Fig.1, crystal fibres can be grown with a wide spectrum of shapes and lengths and they can be used for the design of many calorimeters, either as active material components or as wavelength shifters in a Shashlik calorimeter cell [14].

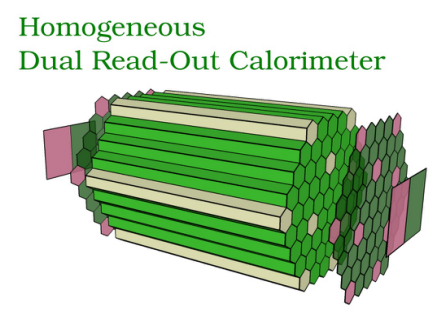

Pointing Fibers

in a Spaghetti Calorimeter

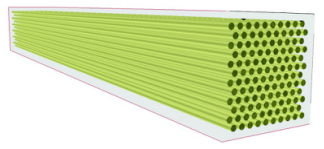

Layers of Crystal Fibers

in a sampling calorimeter
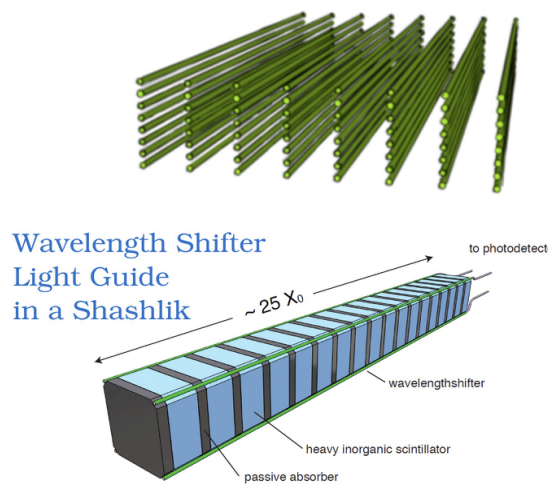

Fig. 1. (Top Left) Simplified drawing of a homogeneous calorimeter providing a high granularity and dual readout capabilities due to the combination of Cherenkov (white) and scintillating (green) fibres. (Top right) Schematic view of crystal fibres planes which can be inserted into an absorber structure. (Bottom left) Example of a SpaCal sampling calorimeter module consisting of a dense absorber filled with crystal fibres with longitudinal axis oriented along beam direction. (Bottom right) Crystal fibres used as a radiation hard wavelength shifter to readout the light from the cells of a Shashlik calorimeter.

In all designs proposed above the light signal can be read-out by grouping together a bunch of fibres (separately for doped and undoped ones) using optical fibres or light guides which collect the photons exiting the fibres and bring it to the photodetector. Such option would allow reducing the number of channels at the cost of a coarser granularity. Alternatively each fibre can be read out independently to maximize the spatial granularity and imaging capability of the detector in reconstructing the topology of hadronic showers and jets. The recent and fast developments in the field of Silicon Photomultipliers (SiPM) represents an attractive option for this type of application as this technology has already been proven to be cost effective, radiation tolerant and capable of operating in high magnetic fields [15].

A variety of electromagnetic and hadronic calorimeter designs were investigated using a Geant 4 simulation tool and results have been summarized in [16]. As reported in Fig.2, energy resolution for charged pions is predicted to be at the level of $22 \% / \sqrt{ } \mathrm{E}$ in single readout for a homogeneous calorimeter made of crystal fibres. 
The resolution improves to about $15 \% / \sqrt{ } \mathrm{E}$ when dual-readout corrections based on the comparison of the scintillation and Cherenkov signal are applied. It was also demonstrated that, for designs which are not fully homogeneous, the energy resolution does not degrade too much provided the calorimeter possess a first homogeneous layer with thickness of about two hadronic interaction lengths $\left(2.15 \lambda_{1}\right)$. This latter point is of high importance as the reduction of the active volume fraction would consequently results in a reduced cost for the final detector.

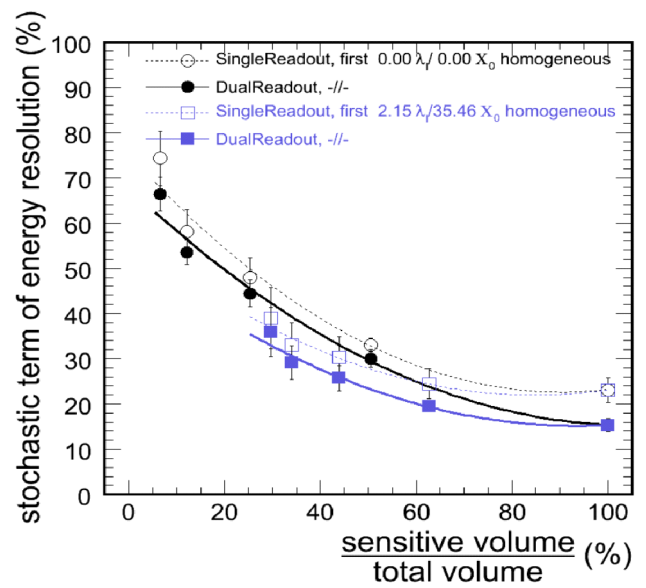

Fig. 2. Simulated performance, in terms of stochastic term of the energy resolution, for single- or dual-readout calorimeters based on LuAG with various sampling configurations of ionisation and Cherenkov signal readout, as described in [16].

A very first measurement of both doped and undoped LuAG crystal fibres of 80 $\mathrm{mm}$ length and $2 \mathrm{~mm}$ diameter was made at CERN using high energy electron beams. This first test beam study allowed measuring the difference between scintillation and Cherenkov pulse shapes and intensity while varying the orientation of the fibres with respect to the beam direction, as shown in Fig.3. Using experimental data as input, simulation studies were also performed to evaluate the optimal fraction of Cherenkov active elements with respect to the full sensitive volume of the detector. 


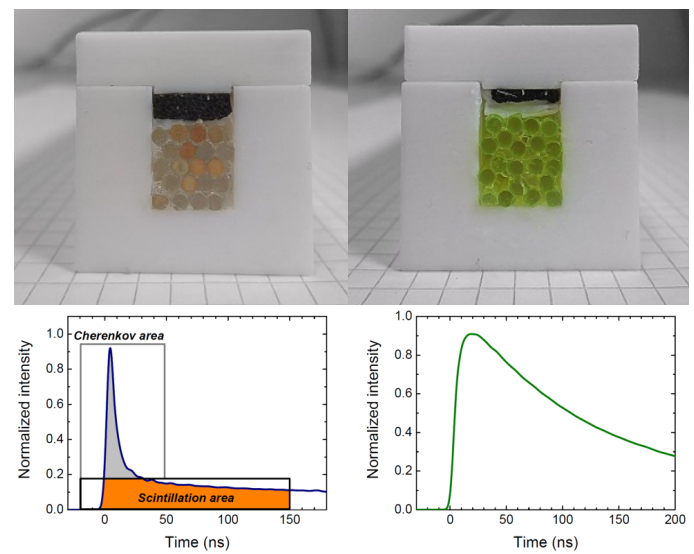

Fig. 3. Pictures of the first units equipped with 80 fibers of undoped LuAG (left) and Ceriumdoped LuAG (right) assembled as a bundle. The plots at the bottom show the typical decay curves obtained for the two units. A small contribution of the exciton-related scintillation of the undoped fibers can be seen.

After these first tests and based on the predictions of the simulation tool, an R\&D project started with the aim of optimizing the properties of crystal fibres for calorimetry applications and to allow the successful construction of larger prototype modules. Some of the key parameters which have been addressed are discussed in the following.

\section{Tailoring crystal fibres for calorimetry}

\section{Attenuation length}

In large calorimeters based on crystal fibres running along the whole detector length, a major obstacle is the light attenuation length $\mathrm{L}_{\text {at }}$ which causes a drop in the signal $\mathrm{I}_{\mathrm{d} \mathrm{d}}$ measured at the photodetector according to:

$$
\mathrm{I}_{\mathrm{dd}}=\mathrm{I}(0) * \exp \left(-\mathrm{z} / \mathrm{L}_{\mathrm{at}}\right)
$$

where $\mathrm{z}$ is the position along the fiber where the light is generated with respect to the location of the photodetector. 
The typical length of electromagnetic calorimeters is around 22 radiation lengths $\mathrm{X}_{0}$ which depends on the density and atomic properties of the calorimeter components and usually ranges between 15 and $30 \mathrm{~cm}$ [14,17]. Lengths of hadronic calorimeters are, on the other hand, much longer and typically range around $10 \lambda_{1}$ (i.e. 1.5-2.5 m) [18]. In order to have a limited impact on the energy resolution of the calorimeter the attenuation length of the fibres should be equal or longer than the detector length. If such condition is satisfied, then the signal does not drop below $1 / \mathrm{e}$ with respect to its original intensity and the spatial non-uniformity of the response does not affect the constant term of energy resolution.

Following a process of optimization of the crystal growth procedure in connection with the material elaboration, the average attenuation length of fibres grown with micro-pulling-down technique has been improved up to $60 \mathrm{~cm}$ (see Fig.4) and the reproducibility required for large mass production was demonstrated [19-20].

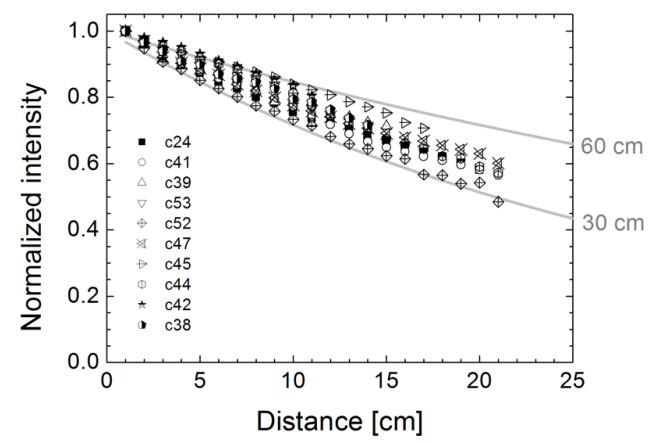

Fig. 4. Attenuation profiles measured on a serie of Ce-doped LuAG fibres grown by the micro-PD technique.

\section{Decay time constants}

In order to be capable of operating in high rates environment, as it would be the case of high luminosity colliders where the time spacing between adjacent bunch crossing can be as short as $25 \mathrm{~ns}$, the decay time constant of the scintillating fibres should not be larger than $40 \mathrm{~ns}$. Since standard Ce-doped LuAG, YAG and GAGG crystals have an average decay time constant higher than $80 \mathrm{~ns}$ their usage at high rates would be limited. However, several studies have been carried out to demonstrate that scintillation kinetics of garnet crystals can be modified by addition of divalent ions, such as $\mathrm{Mg}^{2+}$ or $\mathrm{Ca}^{2+}$ ions [12]. Significant reduction of decay time by factor of 2 have been observed for LuAG:Ce,Mg, YAG:Ce,Mg and GAGG:Ce,Mg 
and have been measured both on bulk crystals and on fibres and are discussed in [12],[21],[22],[23],[24],[25].

The faster decay and the reduction of the intensity of slow radiative recombination processes can be explained by the stabilization of the $\mathrm{Ce}^{++}$centres, due to $\mathrm{Mg}$ co-doping. The $\mathrm{Ce}^{++}$centres provide an alternative channel for fast radiative de-excitation by competing with any kind of electron traps in the material for the capture of an electron from the conduction band. This technique represents a promising way for further optimization of the scintillation mechanisms in crystal fibres.

\section{Radiation tolerance}

The radiation levels that calorimeters have to withstand when operating at high luminosity hadron colliders are extremely high, due to large amount of particles originating from each collision and to the high collision rate. Ionizing radiation (e.g. from energetic electrons and photons) can achieve integrated doses up to $100 \mathrm{kGy}$ during the detector lifetime. Integrated fluences of charged and neutral hadrons can also be as high as $10^{\text {s }}$ particles $/ \mathrm{cm}^{2}$. Both type of radiation are capable of producing different type of defects in the scintillating materials, as well as in the detector electronics, and thus their effect on crystal fibres has to be investigated in detail. In particular, for long fibres where the light has to travel a long path before detection, it is of fundamental importance that the induced light absorption coefficient of the bulk material, $\mu_{\text {ind }}$, is kept below $2 \mathrm{~m}^{-1}$.

Several studies have been performed on all the type of garnet crystals mentioned above to study the effect of high radiation levels on the transparency and scintillation properties of the samples [11]. If samples are grown with a good purity of the raw material and no impurities, they show minimal radiation damage. It has also been observed that divalent ions codoping can further improve the radiation tolerance in garnet scintillators, as in the case of GAGG:Ce,Mg [26], further motivating research efforts in this direction. 


\section{Prototyping and beam tests}

From the initial concept proposed in [7], a variety of pre-prototypes have been assembled and tested in multiple beam test campaigns [27-28]. Both homogeneous and sampling-based designs were considered. Two different orientations of the fibres with respect to the beam direction were also investigated: pointing (fibres pointing to the beam) and transverse (fibres perpendicular to the beam).

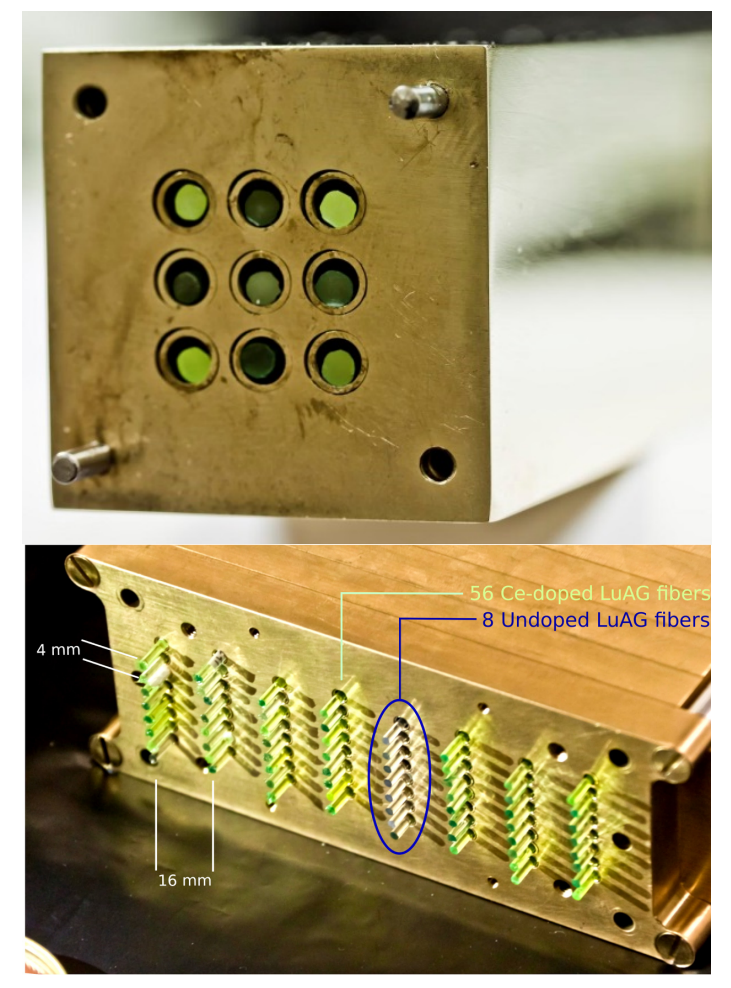

Fig. 5. (Top) LuAG fibres in a unit of brass absorber with pointing geometry (fibers are poiting toward the beam) as discussed in [27] and (bottom) prototype of calorimeter with 64 LuaG fibres in transverse configuration as discussed in [28].

In the beam tests, both doped and undoped LuAG fibres of more than $200 \mathrm{~mm}$ length and $2 \mathrm{~mm}$ in diameter, grown via micro-pulling-down technique, were used. In a first study [27] the light from the fibre was collected using an optical fibers with length of several metres to bring the signal to SiPMs located off the detector. The fibres were embedded in a SpaCal-like brass module, with a spacing of about $3 \mathrm{~mm}$ between adjacent holes, as shown in Fig. 5 (top). 
A good linearity of the signal was measured by using electrons of different energies between 50 and $150 \mathrm{GeV}$ confirming that scintillation mechanism within the fibre is proportional to the energy deposited within the active volume. The energy resolution of the module was however limited by the small sampling fraction $(\sim 10 \%)$ and by the transverse size of the calorimetric cell which did not provide full containment of electromagnetic showers.

A read-out option which makes use of optical fibres is not optimal for light collection as only a small fraction of the photons $(\sim 0.2 \%)$ is detected due to losses at the interface between crystal and optical fibres as well as attenuation losses along the clear fibre. In addition, typical optical fibres are made of plastic materials which suffer a significant transparency drop under irradiation [29] and thus are not suitable for usage in harsh radiation environments.

Another type of prototype assembled consists of an arrangement of crystal fibres in the transverse configuration, as shown in Fig. 5 (bottom). The absorber unit was filled with $56 \mathrm{LuAG}$ :Ce fibres ( 7 rows with 8 fibers in each) and one row of 8 undoped LuAG fibres. All were of $20 \mathrm{~cm}$ length and $2 \mathrm{~mm}$ diameter. This prototype was tested at the Fermilab test beam facility (FTBF) using a mixed beam of electrons and pions with energy between 2 and $16 \mathrm{GeV}$. The light signal exiting from the fibres was read out at one end using an array of Ketek SiPMs directly coupled with optical grease to the crystal, having a photon detection efficiency of $20 \%$ at $520 \mathrm{~nm}$ (emission peak of LuAG:Ce). This configuration allowed a light detection efficiency 10 times better $(\sim 2 \%)$ with respect to previous prototype with a signal up to $15 \mathrm{phe} / \mathrm{MeV}$.

The results obtained during these test beam campaigns [28] are illustrated in Fig.6. All the 64 fibres yielded high signals easily readout by SiPMs. The energy reconstructed from the calorimeter prototype scaled linearly with the beam energy, confirming previous results. In addition, the granularity of this type of calorimeter allows to track the shower development across the different layers of fibres and to separate the incoming particles based on their nature (electrons/pions separation). The latter point is illustrated in Fig.6 (bottom left) where the events are discriminated according to the number of fibres lighting up and the deposited energy. As electrons generate an electromagnetic shower inside the calorimetric module the number of fibres which detect a signal is on average around 40 and the total energy deposit is large. Each layer measures a signal proportional to the intensity of the electromagnetic shower at a given calorimetric depth. Conversely, the pions have a very small probability to generate a shower in such small volume and mostly act as minimum ionizing particles which traverse the module along a straight trajectory. In this case, the number of fibres which detect a signal in correspondence with the passage of a pion is around 8 (i.e. only one fibre in each layer) and the total energy deposit is order of magnitude smaller compared to electrons. These discrimination variables and imaging capability represent a very powerful tool in separating pions from electrons. 
10
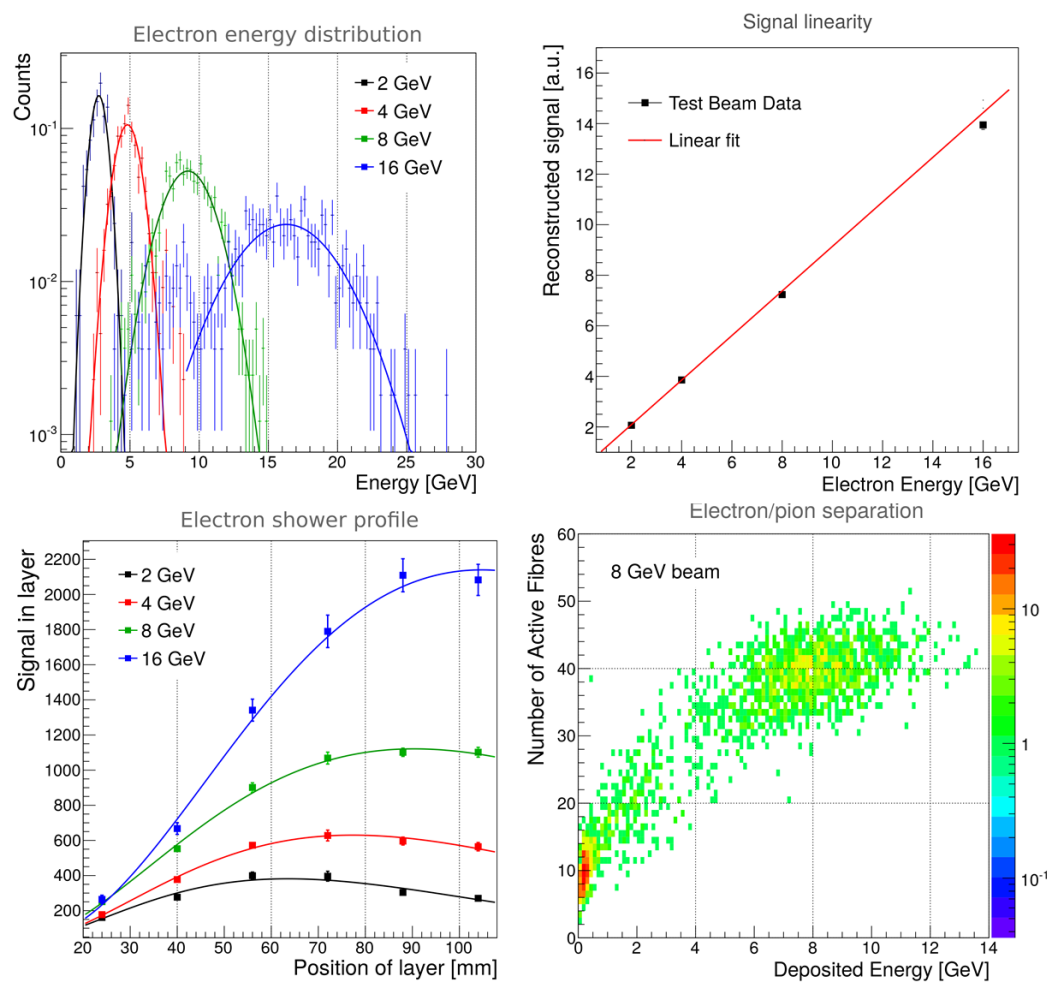

Fig. 6. Test beam results obtained with the brass/LuAG prototype described in Fig. 5 demonstrating the linearity of the calorimeter (top), its ability to track the shower development (bottom left) and identify particles (bottom right).

All the experimental work was also thoroughly compared to Geant 4 predictions allowing the validation of the simulation models. Once validated, the simulation has then been used to investigate the best configurations to be considered for a large scale prototype. The main focus was set at determining the optimal sampling fraction and the fibre disposition within the absorber in order to maintain a good uniformity of the active volume and minimize the constant term of energy resolution.

The prototypes considered so far, although assembled as sampling calorimeters, were having a rather homogeneous overall density since LuAG crystal fibers and the brass absorber did not differ too much in density. Another line of work was focused on the study of calorimeter units built of elements of lower density. For this purpose, a new type of absorber units were designed in Copper-Tungsten alloy and the holes were filled with Yttrium-based garnet crystals (YAG:Ce) and also silica glasses ( $\mathrm{SiO} 2: \mathrm{Ce})$, as illustrated in Fig.7. The advantages of silica glasses are a reduced production cost and fast pulling rates which become of high interest when mass production is to be considered. Optimization of this material is however 
needed to reach similar radiation hardness levels as those achieved in garnet single crystals.

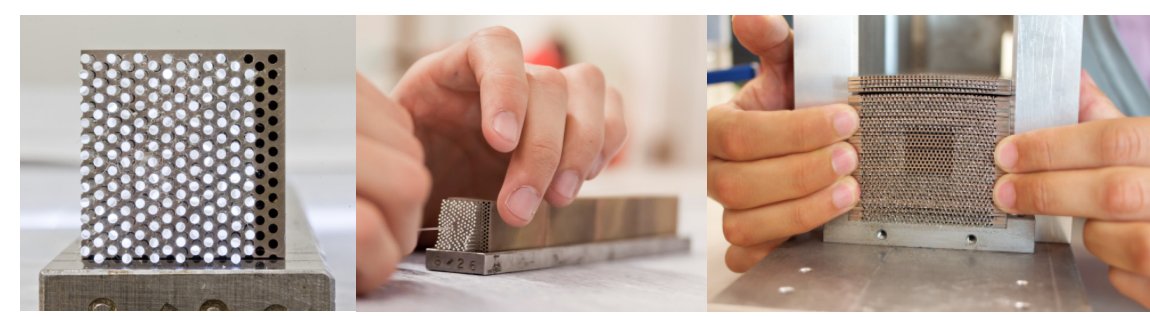

Fig. 7. Tungsten-Copper absorber units for electromagnetic calorimeter designs (left) and larger scale assembly with 9 sub-units. Each unit can be filled with various fibre-shaped materials: Cerium-doped silica glasses (as illustrated here), DSB fibres or also YAG:Ce crystal fibers.

The assembled prototype with the Tungsten-copper absorber can be read with individual PMTs collecting the light from one absorber unit (121 holes). Test beam campaigns are now planned for this summer with units equipped with $\mathrm{SiO} 2$ :Ce and YAG:Ce fibres as well as with commercial plastic scintillators used as reference.

\section{Discussion and outlook}

The crystal fibre technology has significantly improved in the past years and become more and more attractive for HEP detectors. However, more challenges must be addressed in order to face the possibility of constructing calorimeters of large volume. In addition to the further investigation required to be carried out on the radiation hardness and timing capabilities of such materials, a critical issue to address is their production at very large scale. The challenge to overcome is to produce hundreds of kilometres of fibres with consistent quality, high reproducibility and reasonable production costs. Multiple fibres pulling based on multi-nozzle crucibles can for instance be considered for this purpose. R\&D is now going on to provide some preliminary answers and investigate production strategies to address these issues. A consortium of research institute and industrial partners have joint their efforts through the European Union's Horizon 2020 research and innovation (RISE) program Intelum with such objectives in mind. As already mentioned, others materials of lower density are also under investigation. This larger screening of materials is conducted with the aim to identify the most suitable candidates fulfilling the challenging conditions required for the next generation of calorimeters to operate at future colliders. 
Acknowledgments The authors wish to express their gratitude to P. Lecoq, D. Deyrail, N. Siegrist and H. Gerwig (CERN, Switzerland), K. Lebbou and C. Dujardin (ILM, France), to X. Xu (Jiangsu Normal University), to N. Aubry and S. Faraj (Fibercryst, France), to T. Medvedeva and C. Tully (University of Princeton, USA), to A. Heering (University of Notre-Dame, USA), to V. Kononets and O.Sidletskiy (ISMA, Ukraine), to K. Blazek and S. Ochesanu (Crytur, Czech Republic), to M. Korjik (INP-BSU), to N. Chiodini, F. Cova, M. Fasoli, F. Moretti and A. Vedda (University of Milano-Bicocca, Italy), to M. Nikl (Institute of Physics, Czech Republic), to K. Kamada and A. Yoshikawa (C\&A) and more generally all our colleagues from the Crystal Clear collaboration in the scope of which the research was conducted. The authors received funding from the French National Agency for Research under grant agreement ANR-10-BLAN-0947 (INFINHI), from the European Union FP7/2007-2013 under grant agreement 289355-PicoSEC-MCNet and from the European Union's Horizon 2020 research and innovation program under the Marie-Curie grant agreements 644260 (Intelum) and 654168 (AIDA-2020).

\section{References}

[1] The HiLumi LHC Collaboration, HL-LHC Preliminary Design Report, (2014)

[2] T. Behnke et al. The International Linear Collider Technical Design Report (2013)

[3] M. Aicheler et al. A Multi-TeV Linear Collider Based on CLIC Technology: CLIC Conceptual Design Report (2012)

[4] https://fcc.web.cern.ch/Pages/default.aspx

[5] The CALICE collaboration. Journal of Instrumentation 5 (2010) 055004

[6] R. Wigmans. Nucl. Instrum. Methods, A 617 (2010) 1-3 129-133

[7] P. Lecoq. IEEE Nuclear Science Symposium (2008) 1405-1409.

[8] P. Lecoq. Journal of Physics 160 (2009) 112016

[9] E. Auffray et al. IEEE Trans. on Nuclear Science (2010) 57(3):1454-1459

[10] K. Lebbou, Optical Materials (2017) 63 13-18

[11] M. Lucchini et al., IEEE Trans. on Nuclear Science (2016) 63(2):586-590

[12] M. Nikl et al., Crystal Growth \& Design (2014) 14(9) 4827-4833

[13] M.T.Lucchini et al., Nucl. Instrum. Methods, A 852 (2017) 1-9

[14] R. Becker et al., Nucl. Instrum. Methods, A 804 (2015) 79-83

[15] A. Heering et al., Nucl. Instrum. Methods, A 824 (2016) 111-114

[16] G. Mavromanolakis et al., Journal of Instrumentation (2011) 6(10):10012

[17] The CMS Collaboration, The CMS Electromagnetic Calorimeter Project Technical Design Report, 361 CERN/LHCC 97-33, CMS TDR 4, (1997)

[18] The CMS Collaboration, The CMS hadron calorimeter project: Technical Design Report, CERN-LHCC-97-031, (1997).

[19] K. Pauwels et al., Journal of Instrumentation (2013) 8 (09):09019

[20] X. Xu et al., Acta Materialia (2014) 67 232-238

[21] A. Nagura, et al., Japanese Journal of Applied Physics, 54 (2015), p. 04DH17

[22] A. Petrosyan et al., Journal of Crystal Growth, 430 (2015), p. 46

[23] K. Kamada et al., Journal of Crystal Growth, 352 (2012), p. 88

[24] K Kamada et al., Optical Materials, 41 (2015), p. 63

[25] V. Kononets et al. other section of this edited book (Development of YAG:Ce and YAGG:Ce scintillation fibers) (2017).

[26]: M. Lucchini et al., Nucl. Instrum. Methods, A 816 (2016) 176-183

[27] M. Lucchini et al., Journal of Instrumentation (2013) 8 (10) 10017

[28] A. Benaglia et al., Journal of Instrumentation (2016) 11 P05004

[29] V. Khachatryan et al., Journal of Instrumentation (2016) 11 T10004 